\title{
Guest editorial: Special issue on software reliability engineering
}

\author{
Laurie Williams
}

Published online: 27 April 2010

(C) Springer Science+Business Media, LLC 2010

This special issue of the Empirical Software Engineering journal is devoted to four papers selected from the 19th International Symposium on Software Reliability Engineering (ISSRE) that was held in Redmond, Washington on the Microsoft campus in 2008. The papers were selected by the program committee as the best four papers in the conference due to the quality, novelty, and sound empirical analysis of the papers. The authors of the four papers have extended their ISSRE publications to include additional detail and analysis.

The first paper by Libin Zheng and Michael Lyu entitled "An Adaptive QoS-Aware Fault Tolerance Strategy for Web Services" presents an approach for selecting and composing appropriate web services to satisfy non-functional requirements, specifically a weighted average of the desired levels of response time, reliability, and resource usage. The approach is evaluated using six service replicas having different non-functional characteristics along with six different sets of user requirements. The results of this experiment show that the dynamic fault tolerance strategy proposed in the paper performs better than standard retry, recovery block, N-version programming, and active methods.

The second paper, entitled "Testing Peer-to-Peer Systems" by Eduardo Cunha de Almeida, Gerson Sunyé, Yves Le Traon, and Patrick Valduriez proposes a framework and methodology for testing peer-to-peer ( $\mathrm{P} 2 \mathrm{P})$ applications. Using the framework, test cases precisely control the volatility of nodes during their execution. The authors validated the framework through implementation and experimentation on an open-source P2P system. The results of their experimentation indicated that the tests were able to detect complex implementation problems.

The third paper by Constantin Sarbu, Andreas Johansson, Neeraj Suri, and Nachiappan Nagappan entitled "Profiling the Operational Behavior of OS Device Drivers" proposes a methodology to gain insight into the operational profile of operating system device drivers at runtime. Such profiles are used to improve test adequacy against real-world workloads by enabling similarity quantification across them. The profiles also reveal execution hotspots in terms of device driver functionalities activated in the field. The authors performed a case study on Microsoft Windows XP and Vista operating system device drivers using various performance and stability benchmarks as workloads.

L. Williams (ه)

North Carolina State University, Raleigh, NC, USA

e-mail: williams@csc.ncsu.edu 
The fourth paper entitled "Support Planning and Controlling of Early Quality Assurance by Combining Expert Judgment and Defect Data-A Case Study" by Michael Klaes, Haruka Nakao; Frank Elberzhager, and Jürgen Münch received the Best Paper Award at the ISSRE 2008 conference. The paper presents a hybrid method for planning and controlling quality assurance, combining commonly-available measurement data and context-specific expert knowledge. To evaluate the method's applicability and usefulness, the authors conducted a case study in the context of independent verification and validation activities for critical software in the space domain. Their hybrid model improved estimation accuracy.

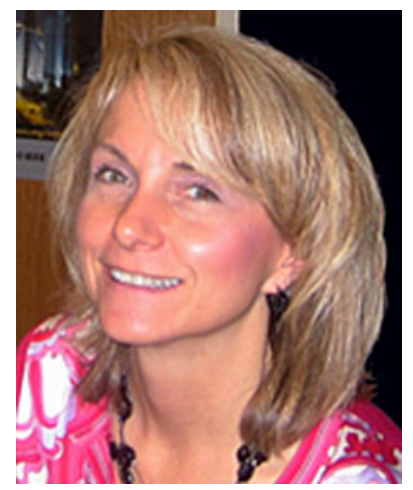

Laurie Williams is an Associate Professor in the Computer Science Department of the College of Engineering at North Carolina State University (NCSU). Her research focuses on agile software development practices and processes; software reliability, software testing and analysis; software security; open source software development; and broadening participation and increasing retention in computer science. Laurie also trains and coaches agile teams. Laurie received her Ph.D. in Computer Science from the University of Utah, her MBA from Duke University Fuqua School of Business, and her BS in Industrial Engineering from Lehigh University. She worked for IBM Corporation for nine years in Raleigh, NC and Research Triangle Park, NC before returning to academia. 